\title{
Resistência ao desgaste e rugosidade superficial de dentes artificiais submetidos ao ensaio de atrição
}

\section{Wear Resistance and Surface Roughness of Artificial Teeth Under Attrition}

\author{
Raissa Ananda Paim Strapasson \\ Faculdade de Odontologia, Universidade Federal do Rio Grande do Sul, Porto Alegre \\ Daniela Maffei Botega \\ Universidade Federal do Rio Grande do Sul \\ Luciana Mayumi Hirakata \\ Faculdade de Odontologia, Pontifícia Universidade Católica do Rio Grande do Sul \\ Juliana BaLbinot HILGERT \\ Faculdade de Odontologia, Universidade Federal do Rio Grande do Sul
}

\begin{abstract}
RESUMO
Objetivo: este estudo avaliou a rugosidade superficial e a resistência ao desgaste de dentes artificiais de resina acrílica submetidos ao ensaio de atrição. Material e métodos: foram selecionados dez incisivos centrais superiores e dez incisivos centrais inferiores de cada marca comercial: Biotone (Dentsply), Trilux (Vipi) e Soluut $\mathrm{PX}$ (Kota). As amostras foram submetidas à aferição de rugosidade superficial em rugosímetro (Mitutoyo SJ-201), com velocidade de $0,5 \mathrm{~mm} / \mathrm{s}$, por trinta segundos e comprimento de leitura de $0,25 \mathrm{~mm}$ e à mensuração da altura cérvico-incisal utilizando paquímetro digital (Mitutoyo Digimatic Caliper), assim como à aferição da massa de cada par de amostras (incisivo superior e inferior) em balança de precisão (Shimadzu AUW220D), antes e após o ensaio. Foi utilizada máquina de escovação mecânica, simulando movimento de atrição entre a superfície vestibular do incisivo superior e a incisal do incisivo inferior. As amostras foram submetidas a 40 mil ciclos, imersas em água destilada. Resultados: não houve diferença estatisticamente significante com relação à rugosidade superficial antes e após o ensaio de atrição. Conclusão: todas as marcas comerciais estudadas apresentaram redução significativa na altura cérvicoincisal; apenas Soluut PX apresentou redução estatística em sua massa. Também se constatou que não houve diferença nos valores de rugosidade entre as marcas comerciais.
\end{abstract}

Palavras-chave: resistência ao desgaste; atrito dentário; rugosidade superficial.

\begin{abstract}
Objetive: This study evaluated the surface roughness and wear resistance of acrylic resin artificial teeth submitted to the test of attrition. Material and Metods: Ten upper central incisors and ten lower central incisors of each brand - Biotone (Dentsply), Trilux (Vipi) and Soluut PX (Kota) - were selected. The samples were subjected to the measurement of surface roughness on a roughness tester (Mitutoyo SJ -201) with $0.5 \mathrm{~mm} / \mathrm{s}$ of speed for 30 seconds and $0.25 \mathrm{~mm}$ of distance; the measurement of cervical-incisal height was performed using a digital caliper (Mitutoyo Digimatic Caliper), as well as the measurement of the weight of each pair of samples (1 upper central and 1 lower central incisors) on a precision scale (Shimadzu AUW220D) before and after the test. A mechanical brushing machine was used to simulate the movement of attrition between the labial surface of the upper incisor and incisal surface of the lower incisor. The samples were subjected to 40.000 cycles of attrition immersed in distilled water. Results: There was no statistically significant difference with respect to surface roughness before and after the test. Conclusion: All trademarks studied showed significant reduction in cervical-incisal height, but showed no significant reduction in their weight, except Soluut PX. Besides that, it was found that there was no difference in roughness between the trademarks.
\end{abstract}

Keywords: wear strength; tooth attrition; surface roughness. 


\section{INTRODUÇÃO}

A especialidade odontológica de prótese dentária oferece alternativas para reposição de elementos dentários perdidos visando à reabilitação funcional (mastigação, fonação) e estética do paciente, com o restabelecimento da dimensão vertical e, dentro do possível, da qualidade de vida. ${ }^{1}$

Na década de 1940, um novo material foi introduzido no mercado: o metacrilato de metila (resina acrílica). Desde então, o metacrilato de metila e a porcelana tornaram-se os materiais mais utilizados na fabricação dos dentes artificiais das próteses dentárias. ${ }^{1,2,3} \mathrm{~A}$ porcelana proporciona maior resistência ao desgaste e maior estabilidade de cor, ${ }^{2}$ porém apresenta desvantagens, como falta de adesão ao material da base da prótese, maior suscetibilidade a fraturas, ruído desagradável durante a mastigação, alta capacidade abrasiva da dentição antagonista e maior dificuldade no ajuste oclusal. Por outro lado, a resina acrílica apresenta vantagens, como boa adesão ao material da base protética, excelente absorção de impacto, facilidade no ajuste oclusal, maior facilidade de manipulação e menor suscetibilidade à fratura; entretanto, sua resistência ao desgaste ao longo do tempo tem sido questionada. .,2,3 $^{1,2}$

A resistência ao desgaste é uma propriedade física de relevância quando se pensa em prótese dentária. Ohlmann et al. ${ }^{4}$ sugerem que o desgaste dos dentes artificiais pode ocorrer por atrição de duas estruturas e/ ou pelo contato destes com alguma substância quimicamente ativa da cavidade oral. O desgaste pode causar, além da diminuição da dimensão vertical de oclusão e a consequente diminuição da eficiência mastigatória, a instabilidade oclusal, o aparecimento de atividades parafuncionais, suporte ineficiente dos dentes posteriores, prejuízo à estética, fadiga dos músculos mastigatórios e desconforto para o paciente. . $^{1,3,5,6,7,8,9}$
De acordo com a literatura, a avaliação da resistência ao desgaste é realizada por meio da associação de testes, como escovação mecânica, atrição com diferentes materiais antagonistas, simulador de mastigação, análise da composição e microdureza da estrutura em questão. ${ }^{3,7,8,9,10,11,12}$

Em termos comerciais, alguns estudos têm avaliado a resistência ao desgaste de diferentes marcas de dentes artificiais disponíveis no mercado. ${ }^{1,2}$ Todavia, ainda não há uma definição sobre qual o melhor material para reforçar o dente artificial de acrílico, qual a melhor prensagem (dupla, tripla, quádrupla) para aprimorar as propriedades destes dentes e qual a melhor marca comercial, visando a um menor desgaste ao longo do tempo. Logo, o objetivo deste estudo foi avaliar a rugosidade $e$ a resistência ao desgaste de dentes artificiais de diferentes marcas comerciais, contendo diferentes substâncias de reforço, por meio do ensaio de atrição, tendo como antagonista o mesmo material do dente avaliado.

\section{Metodologia}

Foram selecionadas três marcas comerciais de dentes artificiais utilizadas rotineiramente na confecção de próteses removíveis: Biotone, Trilux e Soluut PX. As especificações dos materiais utilizados estão presentes na Tabela 1.

\section{Confecção das amostras}

Foram selecionados vinte dentes de cada marca, sendo dez incisivos centrais superiores e dez incisivos centrais inferiores, os quais foram pareados (um incisivo superior e um inferior), constituindo sessenta amostras no total do estudo, divididas em três grupos: 1) Biotone, 2) Trilux e 3) Soluut. 
Tabela 1: Especificações dos dentes artificiais das marcas Biotone, Trilux e Soluut PX

\begin{tabular}{|c|c|c|c|}
\hline Marca comercial & Fabricante & $\begin{array}{l}\text { Composição descrita } \\
\text { pelo fabricante }\end{array}$ & Tipo de prensagem \\
\hline Biotone & $\begin{array}{c}\text { Dentsplay Indústria e } \\
\text { Comércio Ltda., Petrópo- } \\
\text { lis, RJ }\end{array}$ & $\begin{array}{c}\text { RA com ligações cruza- } \\
\text { das }\end{array}$ & Dupla prensagem \\
\hline Trilux & $\begin{array}{c}\text { VIPI Indústria e Comér- } \\
\text { cio Ltda., Pirassununga, } \\
\text { SP }\end{array}$ & $\begin{array}{l}\text { RA com dupla ligação } \\
\text { cruzada; EDMA; OMC }\end{array}$ & Tripla prensagem \\
\hline Soluut PX & $\begin{array}{c}\text { Kotalmports, São Paulo, } \\
\text { SP }\end{array}$ & RA e resina composta & Tripla prensagem \\
\hline
\end{tabular}

Para facilitar o posicionamento dos dentes na máquina de ensaio, eles foram fixados em placas retangulares medindo $20 \mathrm{~mm} \times 20 \mathrm{~mm} x$ $5 \mathrm{~mm}$ para os incisivos superiores e $60 \mathrm{~mm} \times 25$ $\mathrm{mm} \times 5 \mathrm{~mm}$ para os inferiores, confeccionadas em resina acrílica autopolimerizável (JET, Clássico Produtos Odontológicos, São Paulo, $\mathrm{SP})$, sendo os incisivos superiores fixados pela face palatina e os inferiores, pela região cervical.

\section{AfERIÇÃo dA MASSA}

A massa das amostras foi aferida antes e após o ensaio de atrição e, para tanto, os corpos de prova permaneceram em um dessecador contendo sílica, em estufa a $37^{\circ} \mathrm{C}$ (Soc. Fabbe Ltda, Marca Registradora, SP), sendo pesadas semanalmente em balança de precisão (Shimadzu AUW220D) até que houvesse a estabilização de sua massa em gramas.

\section{RugosidADE SUPERFICIAL}

O padrão de rugosidade utilizado foi o $\mathrm{Ra}$, que representa a média das distâncias entre picos a vales do trajeto de aferição. Arugosidade superficial foi avaliada na face vestibular de cinco incisivos superiores de cada marca comercial, selecionados aleatoriamente, em sentido transversal em relação ao longo eixo do dente, utilizando rugosímetro (Mitutoyo, modelo SJ-201) com velocidade de $0,5 \mathrm{~mm} / \mathrm{s}$, por 30 segundos e comprimento de leitura de $0,25 \mathrm{~mm}$.

Para cada corpo de prova foram realizadas três mensurações, antes e após o ensaio de atrição, sendo considerado o valor de rugosidade final em cada uma das etapas a média aritmética das três medições em micrometros.

\section{Aferição dA dimensão}

Os incisivos inferiores receberam pontos de referência e tiveram sua altura aferida antes e após o ensaio de atrição utilizando paquímetro digital (Mitutoyo Digimatic Caliper, Mitutoyo Sul Americana Ltda., São Paulo, SP). Foram realizados dois "sulcos" na face vestibular do dente utilizando lâmina de bisturi n. 12 (Solidor, Lamedid, Barueri, SP, Brasil), um no sentido vertical e outro no sentido horizontal, formando um ponto de intersecção entre eles, sendo este considerado o ponto inicial da medição. O ponto central da face incisal do dente (previamente planificada) foi considerado o ponto final da medição, onde outro "sulco" foi realizado no sentido mésio-distal.

As amostras foram medidas, então, da intersecção das linhas da face vestibular até a linha da face incisal, seguindo uma trajetória 
reta entre um ponto e outro. Foram realizadas três mensurações em cada amostra, sendo a média aritmética dos três valores, em milímetros, considerada a medida final.

\section{ENSAIO DE ATRIÇÃo}

Para o ensaio de atrição, foi utilizada uma máquina para ensaios de escovação mecânica adaptada para receber as amostras. Como o objetivo deste estudo foi realizar a atrição entre as superfícies dos dentes artificiais, a face vestibular dos incisivos superiores e a face incisal dos incisivos inferiores foram planificadas com lixa d'água (100, 400 e 800, Norton Saint-Gobain Abrasivos Ltda., Guarulhos, SP) para propiciar o máximo contato entre elas. Posteriormente, foram polidas com pasta para polimento (Poligloss, TDV, Pomerode, SC) e disco de feltro (Diamond, FGM Produtos Odontológicos, Joinville, SC). Este procedimento foi realizado antes da aferição da massa e da dimensão e após a aferição da rugosidade superficial.

Os corpos de prova contendo os incisivos inferiores foram adaptados em recipientes de acrílico contendo $12 \mathrm{ml}$ de água destilada à temperatura ambiente, simulando a umidade da cavidade oral, e posicionados na parte inferior fixa da máquina. Para cada um destes conjuntos foi fixada, utilizando-se esparadrapo de tecido (Rocitex Esparadrapo impermeável, Cremer, Blumenau, SC), uma amostra correspondente contendo o incisivo superior da mesma marca comercial no braço móvel superior da máquina (Figura 1). Para cada amostra foram realizados 40 mil ciclos, simulando aproximadamente 120 dias de função oral normal, ${ }^{13}$ sendo 250 ciclos por minuto, com deslocamento horizontal de 12 $\mathrm{mm}$.

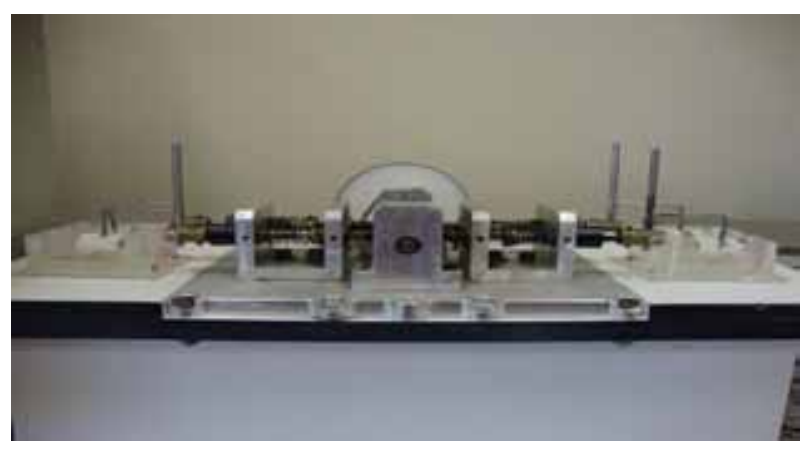

Figura 1 - Amostras posicionadas na máquina para o ensaio de atrição

Os valores obtidos para rugosidade superficial, dimensão cérvico-incisal e massa de cada grupo foram submetidos ao teste $t$ pareado para comparação de cada marca comercial antes e após o ensaio; para a comparação da rugosidade superficial entre os grupos foi realizado o teste de Kruskall-Wallis.

\section{Observação em MEV}

A análise da superfície vestibular de um incisivo superior de cada marca comercial, selecionado aleatoriamente, foi realizada em microscópio eletrônico de varredura (MEV) da Universidade Federal do Rio Grande do Sul (CME_UFRGS modelo JEOL JSM 5800), antes (dente hígido) e após o ensaio de atrição. Previamente à observação, as amostras foram imersas em álcool etílico 99,9\% e mantidas por trinta minutos em ultrassom, para limpeza. Posteriormente, foram retiradas com pinça, secas, metalizadas e guardadas em recipiente plástico, não havendo mais contato manual com elas até o término da observação.

\section{Resultados}

Não houve diferença estatisticamente significativa nos valores de rugosidade superficial entre as marcas avaliadas (Tabela 2). Do mesmo modo, nenhuma marca comercial sofreu alteração significativa de rugosidade antes e após o ensaio de atrição (Tabela 3). 
$\mathrm{Na}$ comparação da dimensão cérvicoincisal antes e após o ensaio de atrição, todas as marcas comerciais apresentaram diferença estatisticamente significativa (Tabela 3). Não houve alteração na massa das amostras dos grupos Biotone e Trilux. Entretanto, o grupo Soluut PX apresentou redução estatisticamente significativa após o ensaio (Tabela 3).
Com relação à observação da superfície dentária em MEV, observou-se a presença de áreas irregulares associadas a poros, antes e após o ensaio de atrição. Entretanto, após o ensaio, nos grupos Biotone (Figura2) e Soluut PX (Figura 3), foi possível identificar estes poros a partir de um aumento de 100x, enquanto no grupo Trilux (Figura 4) estes poros foram identificados a partir de um aumento de 500x.

Tabela 2 - Comparação da rugosidade (média em micrometros e desvio-padrão) entre as marcas Biotone, Trilux e Soluut PX.

\begin{tabular}{cccccc}
\hline Grupo & $\mathbf{n}$ & Pré-ensaio de atrição & $\mathbf{p}$ & Pós-ensaio de atrição & $\mathbf{p}$ \\
\hline Biotone & 5 & $0,222(0,033)$ & 0,49 & $0,316(0,105)$ & 0,58 \\
Trilux & 5 & $0,186(0,043)$ & & $0,280(0,062)$ & \\
Soluut PX & 5 & $0,186(0,023)$ & & $0,260(0,080)$ & \\
\hline
\end{tabular}

Teste de Kruskall-Wallis.

Tabela 3 - Comparação da rugosidade, da altura cérvico-incisal e da massa das marcas Biotone, Trilux e Soluut PX antes e após o ensaio de atrição (média e desvio padrão)

\begin{tabular}{lcccc}
\hline \multicolumn{1}{c}{ Grupo } & $\mathbf{n}$ & Pré-ensaio & Pós-ensaio & $\mathbf{p}$ \\
\hline Rugosidade $(\boldsymbol{\mu m})$ & & & & \\
\hline Biotone & 5 & $0,222(0,033)$ & $0,316(0,105)$ & 0,127 \\
Trilux & 5 & $0,186(0,043)$ & $0,280(0,062)$ & 0,062 \\
Soluut PX & 5 & $0,186(0,023)$ & $0,258(0,079)$ & 0,254 \\
\hline Altura cérvico-incisal $\mathbf{( m m )}$ & & & & \\
\hline Biotone & 10 & $3,256(0,362)$ & $3,124(0,369)$ & 0,001 \\
Trilux & 10 & $2,858(0,497)$ & $2,783(0,497)$ & 0,018 \\
Soluut PX & 10 & $3,046(0,263)$ & $2,948(0,302)$ & 0,017 \\
\hline Massa $(\mathbf{g})$ & & & & \\
\hline Biotone & 10 & $10,654(0,678)$ & $10,530(0,621)$ & 0,150 \\
Trilux & 10 & $10,662(0,748)$ & $10,615(0,724)$ & 0,054 \\
Soluut PX & 10 & $11,121(1,02)$ & $10,556(0,876)$ & 0,029 \\
\hline
\end{tabular}

Teste t pareado. 


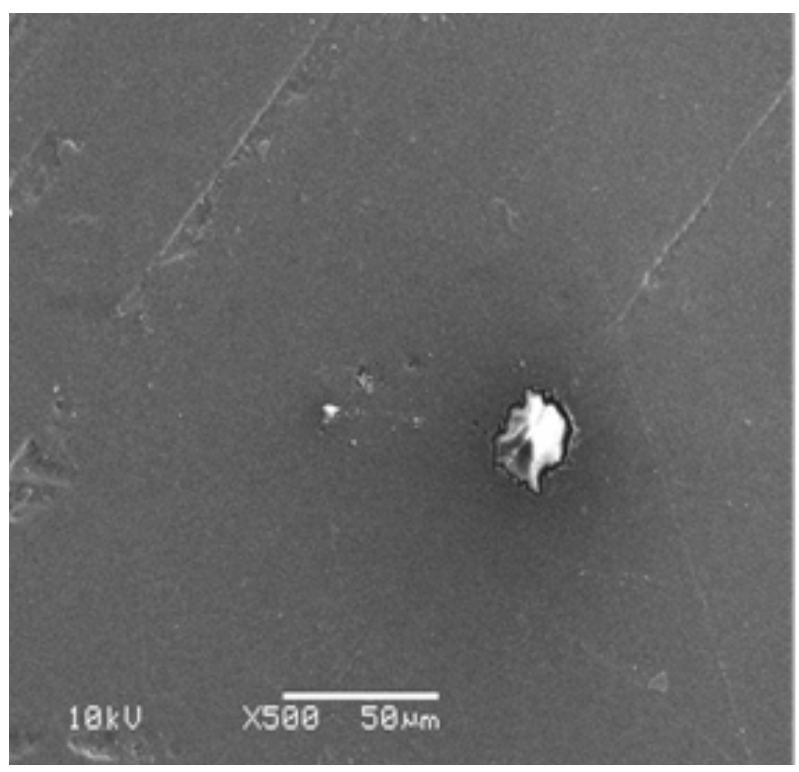

Figura 2 - Imagem em MEV (aumento de 500x) da marca Biotone após o ensaio de atrição

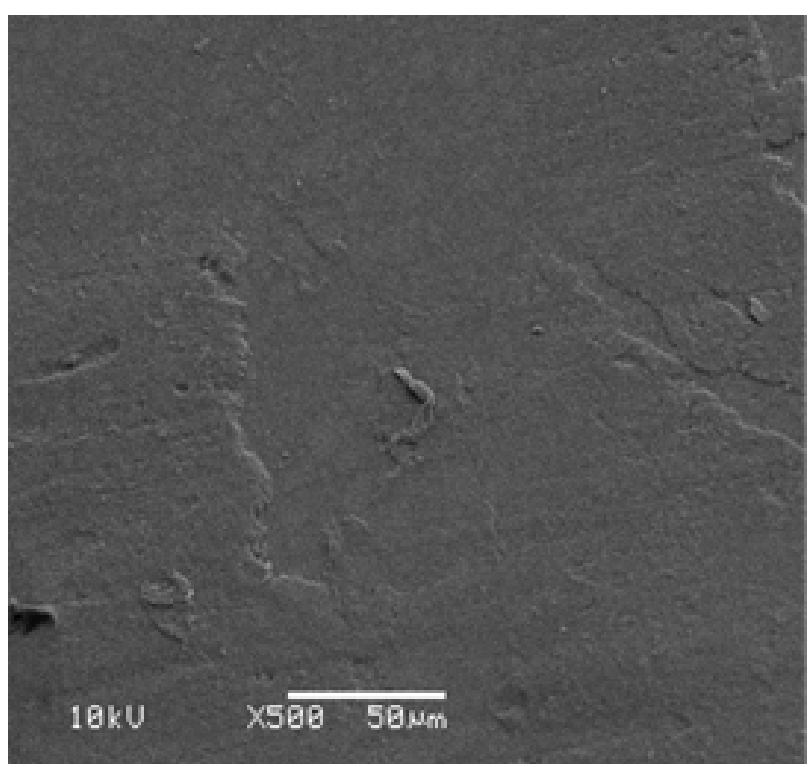

Figura 3 - Imagem em MEV (aumento de 500x) da marca Soluut PX após o ensaio de atrição

\section{Dıscussão}

A rugosidade superficial constitui-se de imperfeições discretas relativamente espaçadas, cuja largura, altura e direção determinam o padrão predominante de uma superfície. ${ }^{14}$ Segundo Oliveira et al., ${ }^{15}$ a rugosidade superficial, quando acima de

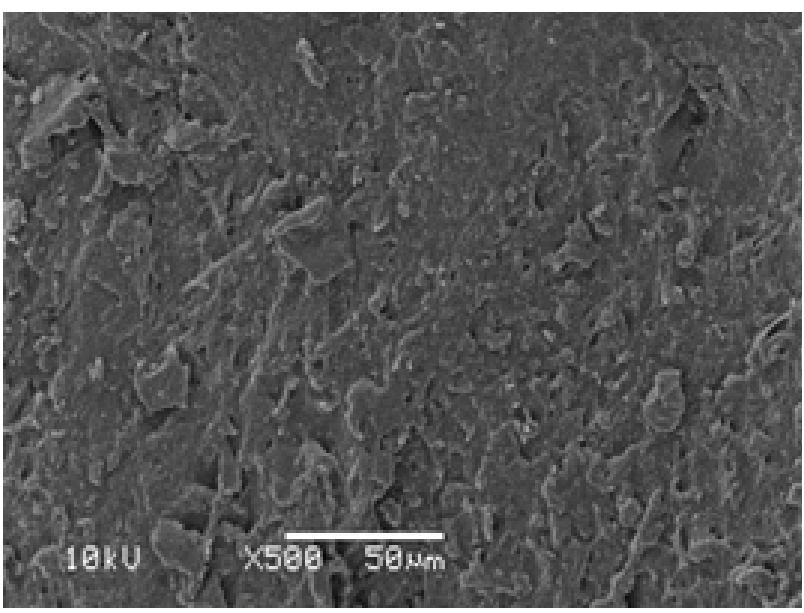

Figura 4 - Imagem em MEV (aumento de 500x) da marca Trilux após o ensaio de atrição

$0,2 \mu \mathrm{m}$, propicia o acúmulo de biofilme e, ao mesmo tempo, esta superfície rugosa protege os microrganismos de serem removidos pela escovação e por forças fisiológicas, como a saliva. Por outro lado, uma superfície lisa reduz a retenção de microrganismos e, consequentemente, previne infecções bucais e a deterioração precoce das próteses. ${ }^{15,16}$

Apesar da diferença na composição dos dentes artificiais, o presente estudo não apresentou divergência nos valores de rugosidade antes e após o ensaio de atrição, tanto na comparação entre os grupos como dentro de cada grupo. Contudo, em ambas as análises, as amostras apresentaram rugosidade superficial acima de 0,2 $\mu \mathrm{m}$ após o ensaio de atrição, sugerindo que, após 40 mil ciclos (120 dias de função oral normal), os dentes artificiais aumentaram sua capacidade de retenção de biofilme. Com relação a esta propriedade, o estudo sugere que dentes de resina acrílica com ligação cruzada, dentes de resina acrílica com dupla ligação cruzada acrescida de dimetilmetacrilato de etilenoglicol (EDMA) polimerizado e de cerâmica organicamente modificada, bem como dentes com resina composta, apresentam o mesmo padrão de comportamento de rugosidade superficial. 
Por meio da observação da superfície em microscópio eletrônico de varredura, o presente estudo mostrou que, antes de serem submetidos ao ensaio de atrição, os dentes artificiais apresentavam poros associados a áreas de irregularidades superficiais, inclusive para o grupo Trilux, cujo fabricante (VIPI Indústria e Comércio Ltda.) afirma ausência total de porosidade. Consideram-se estas irregularidades um padrão de normalidade, já que podem ser atribuídas ao próprio processo de fabricação dos dentes artificiais, que não atinge com perfeição a lisura superficial. Todavia, após os 40 mil ciclos de atrição, apesar da superfície mostrar-se mais regular, os dentes artificiais apresentaram poros nem sempre associados $a$ áreas de irregularidades.

Esta observação não mostrou áreas de ranhuras, como ocorre em alguns trabalhos. Em estudo para avaliar a resistência ao desgaste de dentes artificiais com diferentes antagonistas submetidos à simulação de mastigação, Hahnel et al. ${ }^{9}$ observaram que os dentes com antagonistas artificiais de resina acrílica apresentaram leves ranhuras quando comparados aos dentes com antagonistas de cerâmica. Para estes últimos, o trabalho observa fortes ranhuras. Diferentemente do presente estudo, a análise de Hahnel et al. ${ }^{9}$ não observou a existência de poros nestas superfícies.

Do mesmo modo, Stober et al. ${ }^{12}$ não observaram alterações na porosidade dos dentes artificiais testados. Após serem submetidos ao ensaio em máquina de desgaste, os dentes de resina acrílica e de resina composta apresentaram uma superfície mais áspera que os dentes de porcelana e de esmalte humano, os quais mostraram maior lisura e regularidade superficial após o ensaio.

Para analisar o desgaste das amostras deste estudo, foram utilizados dois métodos: a aferição da altura cérvico-incisal e a mensuração da massa das amostras em balança de precisão. As duas metodologias foram associadas, pois considerou-se que, mesmo com pontos de referência, a aferição da altura cérvico-incisal poderia sofrer alterações em razão de diferentes angulações do paquímetro em relação aos pontos. Além disso, considerando apenas a altura cérvico-incisal, poderia haver uma subnotificação do real desgaste das amostras, já que não estaria sendo considerado o desgaste ocorrido nos dentes incisivos centrais.

Vale ressaltar que este estudo não simula uma situação clínica, tampouco possui o intuito de fazê-lo, já que a ênfase deu-se em avaliar unicamente o comportamento do material. Sendo assim, os dentes selecionados (incisivos centrais superiores e inferiores) não simulam uma situação clínica real de desgaste (face vestibular em atrito com face incisal) e não são dentes majoritariamente envolvidos na dimensão vertical. Contudo, os elementos dentários selecionados e sua posição durante o ensaio de atrição são suficientes para simular uma situação de desgaste do material, podendo-se inferir que os resultados aqui obtidos seriam os mesmos que ocorreriam para molares em situação semelhante.

Quando a altura cérvico-incisal foianalisada, as três marcas comerciais apresentaram desgaste significativo após o ensaio de atrição. Entretanto, esta diferença estatística parece não ser relevante clinicamente a ponto de causar dano ao paciente, uma vez que a diferença numérica de altura pré e pós-ensaio foi inferior a $1 \mathrm{~mm}$ para todas as marcas estudadas. Uma possível explicação para esta diferença pode ser o pequeno número amostral utilizado no presente estudo, apesar de este estar amparado na literatura. 2,3,7,10,11,12 Embora a diferença obtida seja pelo menor número de ciclos em relação aos trabalhos citados, esta se tornaria relevante em maior tempo.

Quando se analisou a massa das amostras antes e após o ensaio, apenas o grupo Soluut 
PX apresentou diminuição significativa, não havendo diferença para as demais marcas. Pode-se observar nos resultados da Tabela 3 que todos os grupos apresentaram diminuição numérica nos valores de massa após o ensaio (Biotone: 0,124 g, Trilux: 0,047 g e Soluut: 0,565 g). Entretanto, apenas o grupo Soluut acusou diferença estatística, muito provavelmente pelo reduzido número de amostras utilizado no estudo, o que resultou em elevado valor de desvio padrão para este grupo. Da mesma forma como discutido anteriormente, esta diferença iria se tornar relevante em maior número de ciclos.

Estes resultados vão ao encontro de Brigagão et al. ${ }^{1}$ Em estudo semelhante, eles avaliaram a resistência ao desgaste de quatro marcas comerciais de dentes artificiais de resina acrílica reforçada, sendo uma delas Trilux, e compararam-nas a dentes de resina acrílica convencional, sendo uma das marcas Biotone. Após 200 mil ciclos em máquina de escovação, constatou-se que não houve distinção significativa entre os grupos.

Da mesma forma, Stober et al. ${ }^{10}$ também investigaram a resistência ao desgaste de dentes artificiais compostos por diferentes materiais, sendo um deles a resina acrílica. Após 100 mil ciclos em simulador de mastigação, os dentes artificiais não apresentaram discrepância significativa entre si com relação ao desgaste.

O presente estudo não avaliou a influência do material do denteantagonistanodesgaste destes dentes artificiais, o que poderia ter influenciado a taxa de desgaste encontrada. ${ }^{2,4,8,9,13} \mathrm{Em}$ estudo semelhante, Hahnel et al. ${ }^{9}$ investigaram a influência do aço, da esteatite e da resina de dentes antagonistas no desgaste vertical e volumétrico de dentes artificiais de resina acrílica. Os achados mostraram que o material do dente antagonista possui grande influência sobre o desgaste do dente artificial, sendo que a esteatite (mineral de baixa dureza) desgasta mais o dente artificial.
Já Schwap et al. ${ }^{17}$ quantificaram o desgaste de dentes artificiais compostos somente por resina acrílica e por resina acrílica acrescida de EDMA in vivo, avaliando também as variações individuais sobre este desgaste durante um ano de acompanhamento. Ao final do estudo, não foram encontradas diferenças significativas no desgaste vertical e volumétrico dos materiais analisados. Notou-se, porém, que com o aumento da idade há também aumento do desgaste dos dentes posteriores. Como o presente estudo foi realizado in vitro, não foram levadas em consideração estas variações individuais que podem influenciar a taxa de desgaste.

Seguindo esta mesma linha, Ohlmann et al. ${ }^{4}$ avaliaram a influência do gênero, do material do dente antagonista e do tipo de prótese no desgaste de dentes artificiais. Como resultado, a análise mostrou maior desgaste dentário em pacientes do gênero masculino. Além disso, o estudo mostrou que usuários de prótese total apresentam menor desgaste do que usuários de prótese parcial, na medida em que pacientes edêntulos tendem a preferir alimentos mais macios e pastosos. Por fim, o estudo mostrou que dentes artificiais em contato com dentes naturais apresentam maior desgaste.

Para Schwap et al., ${ }^{17}$ espera-se que as taxas de desgaste dentário sejam maiores em pacientes edêntulos do que em pacientes dentados, em função da ausência dos receptores periodontais responsáveis por controlar a força oclusal. Estudos clínicos utilizando eletromiografia mostram que pacientes edêntulos apresentam maior atividade muscular e necessitam de mais ciclos mastigatórios do que aqueles que possuem dentes naturais. Associadas, estas condições poderiam explicar este maior desgaste. ${ }^{18}$

No presente estudo foi avaliado o desgaste de dentes artificiais de resina acrílica a partir de antagonistas do mesmo material. Porém, se o antagonista utilizado fosse um dente natural, 
os resultados poderiam ter apresentado um desgaste significativo, considerando os achados de Ohlmann et al. ${ }^{4}$ Também se pode supor que, se este estudo ocorresse in vivo, o desgaste encontrado poderia ser ainda maior, já que outros fatores estariam atuando sobre estes dentes - hábitos parafuncionais, tipo de alimento ingerido, tipo de escova utilizada para higienizar a prótese, força de escovação, tipo de prótese utilizado, número de dentes naturais presentes na boca, contatos oclusais. . $^{2,4,17,19}$

Dessa forma, com base nos resultados encontrados, o que deve guiar o clínico na decisão sobre qual marca comercial utilizar é a adaptação do profissional a cada uma delas, a estética que cada uma pode proporcionar e o custo de cada marca. A escolha por Biotone, Trilux ou Soluut $\mathrm{PX}$ baseada na resistência ao desgaste, na rugosidade ou na dureza não se justifica. Do mesmo modo, a diferença de preço entre elas não pode ser explicada por estes aspectos.

\section{Conclusões}

As marcas Biotone, Trilux e Soluut PX apresentaram o mesmo padrão de comportamento de rugosidade superficial antes e após o ensaio de atrição, sem diferença significativa; com relação à massa, apenas a marca Soluut PX apresentou redução estatística após o ensaio.

As três marcas apresentaram o mesmo padrão de comportamento em relação à altura cérvico-incisal, havendo diminuição significativa após o ensaio.

Estas informações são de fundamental importância no momento da escolha da marca comercial de dentes artificiais na prática clínica. Considerando-se os aspectos analisados e o contexto social dos pacientes em atendimento odontológico nas universidades, a marca Biotone seria a mais indicada.

\section{REFERÊNCIAS}

1 Brigagão VC, Camargo FP, Neisser MP. Avaliação in vitro da resistência ao desgaste de dentes artificiais. Cienc Odontol Bras. 2005 Jul.Sep.; 8(3): 55-63.

2 Hirano S, Kenneth BM, Warren CW, Corrine $\mathrm{HH}$. In vitro wear of resin denture teeth. J Prosthet Dent. 1998 Feb.; 79(2): 152-5.

3 Ghazal M, Yang B, Ludwig K, Kern M. Two-body wear of resin and ceramic denture teeth in comparison to human enamel. Dent Mater. 2008 Apr.; 24(4): 502-7.

4 Ohlmann B, Rohstock K, Kugler J, Gilde H, Dreyhaupt J, Stober, T. Influences of clinical wear of acrylic denture teeth: a pilot study. Int $\mathrm{J}$ Prosthodont. 2007 Sep.-Oct.; 20(5): 496-8.

5 Ogle RE, Davis EL. Clinical wear study of three commercially available artificial tooth materials: Thirty-six month results. J Prosthet Dent. 1998 Feb.; 79(2): 145-51.

6 Assunção WG, Barbosa DB, Goiato MC, Tabata $\mathrm{LF}$, Genari Filho H. Evaluation of the wear rates of eight different brands of artificial resin teeth. Cienc Odontol Bras. 2005 Jul.-Sep.; 8(4): 6-12.

7 Zeng J, Sato Y, Ohkubo C, Hosoi T. In vitro wear resistance of three types of compositors in denture teeth. J Prosthet Dent. 2005 Nov.; 94(5): 453-7.

8 Reis KR, Bonfante G, Pegoraro LF, Conti PCR, Oliveira PCG, Kaizer OB. In vitro wear resistance of three types of polymethylmethacrylate denture teeth. J Appl Oral Sci. 2008 May.Jun.;16(3): 176-80.

9 Hahnel S, Behr M, Handel G, Rosentritt M. Twobody wear of artificial acrylic and composite resin teeth in relation to antagonist material. J Prosthet Dent. 2009 Apr.; 101(4): 269-78.

10 Stober T, Lutz T, Gilde H, Rammelsberg P. Wear of resin denture teeth by two-body contact. Dent Mater. 2006 Mar.; 22(3): 243-9.

11 Loyaga-Rendon PG, Takahashi H, Hayakawa I, Iwasaki N. Compositional characteristics and hardness of acrylic and composite resin artificial teeth. J Prosthet Dent. 2007 Aug.; 98(2): 141-9.

12 Stober T, Henninger M, Schmitter M, Pritsch M, Rammelsberg $P$. Three-body wear of resin den- 
ture teeth with and without nanofillers. J Prosthet Dent. 2010 Feb.; 103(2): 108-17.

13 Mello PC, Coppedê AR, Macedo AP, Mattos MGC, Rodrigues RCS, Ribeiro RF. Abrasion wear resistance of different artificial teeth opposed to metal and composite antagonists. J Appl Oral Sci. 2009 Sep.-Oct.; 17(5): 451-6.

14 Anusavice KJ. Phillips: materiais dentários. 11a ed. Rio de Janeiro: Elsevier; 2005.

15 Oliveira LV, Mesquita MF, Henriques GEP,Consani RLX. The effect of brushing on surface roughness of denture lining materials. J. Prosthodont. 2007 May.-Jun.; 16(3): 179-84.

16 Gonçalves LFF, Neto DRS, Bonan RF, Carlo HL, Batista AUD. Higienização de próteses totais e parciais removíveis. $\mathrm{R}$ Bras Cienc Saúde. 2011; 15(1): 87-94.
17 Schwap MS,Rousson V,Vornwagner K,Heintze $\mathrm{SD}$. Wear of two artificial tooth materials in vivo: A 12-month pilot study. J Prosthet Dent. 2009 Aug.; 102(2): 104-14.

18 Fontijn-Tekamp FA, Slagter AP, Van Der Bilt A, Van'tHof MA, Kalk W, Jansen JA. Swalowing thresholds of mandibular implant-retained overdentures with variable portion sizes. Clin Oral Implants Res. 2004 Jun.; 15(3): 375-80.

19 Ogle RE, Davis EL. Clinical wear study of three commercially available artificial tooth materials: Thirty-sixmonth results. J Prosthet Dent. 1998 Feb.; 79(2): 145-51.

Submetido em: 26-11-2014

Aceito em: 29-1-2015 